CCNY-HEP 2/94

March 1994

\title{
Hamiltonian Analysis of the Effective Action for Hard Thermal Loops in QCD
}

\author{
V.P. NAIR \\ Physics Department \\ City College of the City University of New York \\ New York, New York 10031.
}

\begin{abstract}
The effective action for hard thermal loops in Quantum Chromodynamics (QCD) is related to a gauged Wess-Zumino-Novikov-Witten theory. Some of the technical issues of this approach are clarified and the Hamiltonian formulation is presented. The two-point correlator for the induced current in QCD is obtained; some simplifications of the dynamics of the longitudinal modes are also pointed out.
\end{abstract}




\section{Introduction}

The possibility of obtaining the quark-gluon plasma phase of hadronic matter in heavy ion collisions or perhaps in certain astrophysical contexts provides an adequate physical motivation for studying Quantum Chromodynamics (QCD) at high temperatures. In a perturbative analysis, hard thermal loops have to be calculated in order to carry out the resummation or rearrangement of thermal perturbation theory necessary to achieve a consistent expansion in powers of the coupling constant. Hard thermal loops are also part of the effective action for the long wavelength excitations of the plasma. They have therefore been the focus of many recent investigations [1-6].

The generating functional $\Gamma[A]$ for the hard thermal loops is a gauge-invariant nonlocal functional of the gauge potential $A_{\mu}$ and is essentially an electric mass term for the gluons. The current given by $\Gamma[A]$ leads to a gauge-invariant description of Debye screening and Landau damping effects $[3]$. $\Gamma[A]$ also has an elegant mathematical structure, being closely related to the eikonal for a Chern-Simons theory [2]. The equations of motion, incorporating contributions from $\Gamma[A]$ are nonlocal, but it is possible to rewrite them as local, but coupled, equations [3]. Further, one can introduce an auxiliary field $G$, which has values in the color group, and an action $\Gamma[A, G]$ which gives the local equations of motion [5]. The elimination of $G$ leads back to the nonlocal functional $\Gamma[A] . \quad \Gamma[A, G]$ is related to the gauged Wess-Zumino-Novikov-Witten (WZNW) theory. A useful result which emerged in a rather simple way within this formulation is the positivity of the Hamiltonian in the hard thermal loop approximation. Actually, one can go further. The local nature of $\Gamma[A, G]$ makes it a convenient starting point for the inclusion of hard thermal loop effects in the systematic calculation of any physical quantity. With this in mind, in this paper, we shall carry out the Hamiltonian analysis for this effective action. This will lead to a current algebraic description of hard thermal loops. A simple framework for the computation of current correlations will also emerge from this analysis. The two-point correlation function for the induced currents in the hard thermal loop approximation, which is related to the gluonic structure functions of the plasma, is obtained to the lowest order. We also point out some simplifications for the dynamics of the longitudinal excitations. 
In section II, we give a brief resume of the effective action, set up the Hamiltonian analysis and obtain the operator algebra. In section III, the framework for computing the correlators and the simplifications for the longitudinal modes are discussed. There are three appendices; the first discusses the issue of whether the coefficient of the effective action should be quantized, the second with the definition of the Hamiltonian and the third with canonical derivation of the operator algebra.

\section{The Effective Action, Hamiltonian and Current Algebra}

The gauge potential $A_{\mu}=\left(-i t^{a}\right) A_{\mu}^{a}$, where $t^{a}$ are hermitian $N \times N$-matrices representing the generators of the Lie algebra of the color group; we take the color group to be $S U(N)$. In addition to the gauge potential $A_{\mu}$, we need the auxiliary field $G(x, \vec{Q})$. This field is an $S U(N)$-matrix depending on spacetime coordinates $x^{\mu}$ and also on a unit three-dimensional vector $\vec{Q}$. Further, $G$ satisfies the condition $G(x,-\vec{Q})=G^{\dagger}(x, \vec{Q})$. We shall also use the notation $Q^{\mu}=(1, \vec{Q}), \quad Q^{\prime \mu}=(1,-\vec{Q})$, and $A_{+}=\frac{1}{2} A \cdot Q, \quad A_{-}=\frac{1}{2} A \cdot Q^{\prime}$. The effective action can be written as [5]

$$
\begin{gathered}
\mathcal{S}=\int-\frac{1}{4} F^{2}+k \Gamma[A, G] \\
\Gamma[A, G]=\int d \Omega\left[\int d^{2} x^{T} S(G)+\frac{1}{\pi} \int d^{4} x \operatorname{Tr}\left(G^{-1} \partial_{-} G A_{+}\right.\right. \\
\left.\left.-A_{-} \partial_{+} G G^{-1}+A_{+} G^{-1} A_{-} G-A_{+} A_{-}\right)\right]
\end{gathered}
$$

where the integration indicated by $d \Omega$ is over all orientations of the unit vector $\vec{Q} . S(G)$ is the WZNW-action for $G$, given by [7]

$$
S(G)=-\frac{1}{2 \pi} \int_{M^{2}} d^{2} x \operatorname{Tr}\left(\partial_{+} G G^{-1} \partial_{-} G G^{-1}\right)+\frac{1}{12 \pi} \int_{M^{3}} \operatorname{Tr}\left(d G G^{-1}\right)^{3}
$$

The integration in the first term is over the lightcone $M^{2}$ defined by the variables $x^{+}=$ $x_{\mu} Q^{\mu}, x^{-}=x_{\mu} Q^{\prime \mu}$; the second term, the so-called Wess-Zumino (WZ) term, requires extension of the fields to include one more coordinate, say $s$, and corresponding integration. We can take $M^{3}=M^{2} \times[0,1]$ with fields at $s=1$ corresponding to spacetime. Different ways of extending the fields to $s \neq 1$ will give the same physical results. Since $S(G)$ has 
integration over the lightcone variables, the integration for $S(G)$ in equation (2) is over the transverse coordinates $x^{T}, x^{T} \cdot \vec{Q}=0 . \Gamma[A, G]$ is gauge invariant and, if we ignore the dependence on the transverse coordinates, is just the standard gauged WZNW-action. Finally the constant $k$ in equation $(1)$ is $\left(N+\frac{1}{2} N_{F}\right) \frac{T^{2}}{6}$, where $N_{F}$ is the number of quark flavors and $T$ is the temperature. The coupling constant $g$ is not explicitly indicated; it can be recovered by the replacement $A_{\mu} \rightarrow g A_{\mu}$.

The WZNW-action $S(G)$ obeys the combination rule

$$
S(h G)=S(h)+S(G)-\frac{1}{\pi} \int_{M^{2}} \operatorname{Tr}\left(h^{-1} \partial_{-} h \partial_{+} G G^{-1}\right)
$$

Using this rule, the equations of motion can be easily derived as

$$
\begin{gathered}
\partial_{+} \mathcal{A}_{-}-\partial_{-} A_{+}+\left[A_{+}, \mathcal{A}_{-}\right]=0 \\
\left(D_{\mu} F^{\mu \nu}\right)^{a}-J^{\nu a}=0 \\
J^{\nu a}=-\frac{k}{2 \pi} \int d \Omega \operatorname{Tr}\left\{\left(-i t^{a}\right)\left[\left(\mathcal{A}_{-}-A_{-}\right) Q^{\nu}+\left(\mathcal{A}_{+}-A_{+}\right) Q^{\prime \nu}\right]\right\} \\
=-\frac{k}{2 \pi} \int d \Omega \operatorname{Tr}\left\{\left(-i t^{a}\right)\left[G^{-1} D_{-} G Q^{\nu}-D_{+} G G^{-1} Q^{\prime \nu}\right]\right\}
\end{gathered}
$$

$D_{\mu}$ denotes the covariant derivative, $D_{\mu}^{a c}=\partial_{\mu} \delta^{a c}+f^{a b c} A_{\mu}^{b}$ and $\mathcal{A}_{+}=G A_{+} G^{-1}-$ $\partial_{+} G G^{-1}, \quad \mathcal{A}_{-}=G^{-1} A_{-} G+G^{-1} \partial_{-} G$.

The current given by equation (7) agrees with what is obtained by evaluation of hard thermal loops, once the solution for $\mathcal{A}_{ \pm}$from equation (5) is substituted in. The action (2) is thus an acceptable effective action at least at the level of the equations of motion. Actually one can make this a stronger statement. The equation of motion for the field $G$ has no independent solutions. This can be seen as follows. We can parametrize the potentials $A_{ \pm}$in terms an $S U(N)$-matrix $V(x, \vec{Q})$ as

$$
A_{+}=-\partial_{+} V V^{-1}, \quad A_{-}=-\partial_{-} V^{\prime} V^{\prime-1}
$$

where $V^{\prime}(x, \vec{Q})=V(x,-\vec{Q})$. The general solution to equation (5) is then given by

$$
G(x, \vec{Q})=V(x,-\vec{Q}) B\left(x^{+}, x^{T}, \vec{Q}\right) C\left(x^{-}, x^{T}, \vec{Q}\right) V^{-1}(x, \vec{Q})
$$


where $C$ is an arbitrary $S U(N)$-matrix depending on the variables indicated and $B$ is given by $C$ with $\vec{Q} \rightarrow-\vec{Q}$. The matrices $B, C$ represent the new or independent degrees of freedom for the field $G$. Notice, however, that the parametrization (8) of the potentials has redundant variables; a transformation $V \rightarrow V U\left(x^{-}, x^{T}, \vec{Q}\right)$, with a corresponding change in $V^{\prime}$, leaves the potentials invariant. Further, ordinary gauge transformations act on the matrix $V$ as $V \rightarrow h(x) V$. Thus the physical subspace for the components $A_{ \pm}$is given in terms of the matrices $V$ with the identifications

$$
V(x, \vec{Q}) \sim h(x) V(x, \vec{Q}) U\left(x^{-}, x^{T}, \vec{Q}\right)
$$

The gauge freedom of multiplying $V$ 's by matrices which do not depend on $x^{+}$, viz. $U$ 's in (10), shows that the we can reduce $G$ to just $V(x, \vec{Q}) V^{-1}(x, \vec{Q})$. There are thus no new real dynamical degrees of freedom in $G$. The action can be simplified for this $G$ to

$$
\Gamma[A, G]=-\int d \Omega d^{2} x^{T} S\left(V^{-1}(x,-\vec{Q}) V(x, \vec{Q})\right)
$$

This agrees with the expression obtained by Feynman graph evaluation of the hard thermal loops.

The Hamiltonian for the effective action (2) was given in ref.[5] as

$$
\mathcal{H}=\int d^{3} x\left\{\frac{E^{2}+B^{2}}{2}+\frac{k}{8 \pi} \int d \Omega \operatorname{Tr}\left[\left(D_{0} G D_{0} G^{-1}\right)+\left(\vec{Q} \cdot \vec{D} G \vec{Q} \cdot \vec{D} G^{-1}\right]-A_{0}^{a} \mathcal{G}^{a}\right\}\right.
$$

where $F_{0 i}^{a}=E_{i}^{a}, F_{i j}^{a}=\epsilon_{i j k} B_{k}^{a}$ and

$$
\mathcal{G}^{a}=(\vec{D} \cdot \vec{E})^{a}+\frac{k}{2 \pi} \int d \Omega \operatorname{Tr}\left[\left(-i t^{a}\right)\left(G^{-1} D_{-} G-D_{+} G G^{-1}\right)\right]
$$

$\mathcal{G}^{a}=0$ is the Gauss law of the theory; it is also the time component of the equation of motion for the gauge field. Expression (12) makes it clear that the Hamiltonian is positive for all configurations which are physical, i.e. obey the Gauss law. (It may be worth recalling that, even for the Maxwell theory, the canonical Hamiltonian is positive only for physical configurations.)

The Hamiltonian analysis is most easily carried out, as in the usual cases, in the gauge $A_{0}^{a}=0$. We start by defining the currents

$$
J_{+}=\frac{k}{4 \pi} D_{+} G G^{-1}=\left(-i t^{a}\right) J_{+}^{a}
$$




$$
J_{-}=-\frac{k}{4 \pi} G^{-1} D_{-} G=\left(-i t^{a}\right) J_{-}^{a}
$$

By virtue of the property $G^{-1}(x, \vec{Q})=G(x,-\vec{Q})$, these are related by $J_{+}(x,-\vec{Q})=$ $J_{-}(x, \vec{Q})$. The Hamiltonian can be written in terms of these currents as

$$
\mathcal{H}=\int d^{3} x\left\{\frac{E^{2}+B^{2}}{2}+\frac{2 \pi}{k} \int d \Omega\left(J_{+}^{a} J_{+}^{a}+J_{-}^{a} J_{-}^{a}\right)\right\}
$$

We have chosen the $A_{0}^{a}=0$ gauge; Gauss law must henceforth be imposed as a constraint. ( For fixed $\vec{Q}$, the integrand of the second term involving the square of $J_{+}$and the square of $J_{-}$is the Sugawara form of the Hamiltonian, well-known for two-dimensional current algebras.) The equations of motion in the $A_{0}^{a}=0$ gauge are

$$
\begin{aligned}
E_{i}^{a} & =\partial_{0} A_{i}^{a} \\
\partial_{0} E_{i}^{a}+\epsilon_{i j k}\left(D_{j} B_{k}\right)^{a} & =\int d \Omega Q_{i}\left(J_{+}^{a}-J_{-}^{a}\right) \\
\left(D_{-} J_{+}\right)^{a} & =-\frac{k}{8 \pi} E_{i}^{a} Q_{i}
\end{aligned}
$$

Equation (16a) is just the definition of the electric field; however, in a Hamiltonian approach it is an equation of motion and we have displayed it as such.

The commutation rules must be such that equations (16) follow as the Heisenberg equations of motion for the Hamiltonian (15). Knowing the current algebra of the WZNWtheory, we can make a guess as to what the appropriate commutation rules are for our problem and verify them by checking that they lead to equations (16) starting from the Hamiltonian (15). The commutation rules are then seen to be

$$
\begin{aligned}
{\left[E_{i}^{a}(\vec{x}), A_{j}^{b}\left(\vec{x}^{\prime}\right)\right] } & =-i \delta^{a b} \delta_{i j} \delta\left(\vec{x}-\vec{x}^{\prime}\right) \\
{\left[E_{i}^{a}(\vec{x}), J_{ \pm}^{b}\left(\vec{x}^{\prime}\right)\right] } & = \pm i \frac{k}{4 \pi} Q_{i} \delta^{a b} \delta\left(\vec{x}-\vec{x}^{\prime}\right) \\
{\left[J_{ \pm}^{a}(\vec{x}, \vec{Q}), J_{ \pm}^{b}\left(\vec{x}^{\prime}, \vec{Q}^{\prime}\right)\right] } & =i f^{a b c} J_{ \pm}^{c} \delta\left(\vec{x}-\vec{x}^{\prime}\right) \delta\left(\vec{Q}, \vec{Q}^{\prime}\right) \\
& \mp \frac{k}{4 \pi} Q_{i}\left(D_{x}\right)_{i}^{a b} \delta\left(\vec{x}-\vec{x}^{\prime}\right) \delta\left(\vec{Q}, \vec{Q}^{\prime}\right) \\
{\left[J_{+}^{a}(\vec{x}, \vec{Q}), J_{-}^{b}\left(\vec{x}^{\prime}, \vec{Q}^{\prime}\right)\right] } & =0
\end{aligned}
$$

All other commutators vanish. $\delta\left(\vec{Q}, \vec{Q}^{\prime}\right)$ stands for the $\delta$-function on the two-dimensional sphere corresponding to the unit vector $\vec{Q}$, i.e. $\int d \Omega_{\vec{Q}^{\prime}} \delta\left(\vec{Q}, \vec{Q}^{\prime}\right) f\left(\vec{Q}^{\prime}\right)=f(\vec{Q})$. 
We have checked that these rules obey the Jacobi identity. Since we are postulating the algebra, this is a necessary check. Of course, commutation rules can be obtained from the action by standard quantization procedures. We have verified that equations (17) follow from such a canonical approach as well. The canonical method is somewhat involved. The most efficient way is to use the language of symplectic structures. This is sketched in the appendix. Finally, the condition $J_{+}^{a}(x-\vec{Q})-J_{-}^{a}(x, \vec{Q})=0$ has to be imposed as a constraint, just like the Gauss law.

We now turn to deriving an equation for the correlators of currents. The parameter $k$ in equation (17c) controls the semiclassical expansion, large $k$ being the classical limit [7]. For a semiclassical expansion, it is convenient to use the currents $I_{ \pm}=\sqrt{\frac{4 \pi}{k}} J_{ \pm}$. Eventhough we are not doing a semiclassical expansion at this stage, it is useful to consider correlators of $I_{+}$'s. ( $I_{-}$'s are related by $\left.\vec{Q} \rightarrow \vec{Q}^{\prime}\right)$. Thus introducing a source $\alpha^{a}(x, \vec{Q})$ for $I_{+}^{a}(x, \vec{Q})$ and a source $\eta_{i}^{a}(x)$ for $A_{i}^{a}(x)$, we can define a generating functional for correlators

$$
Z[\eta, \alpha]=\exp (W[\eta, \alpha])=\left\langle T \exp \left[i \int d^{4} x\left\{\left(\int d \Omega \alpha^{a} I_{+}^{a}\right)+\eta_{i}^{a} A_{i}^{a}\right\}\right]\right\rangle
$$

$W[\eta, \alpha]$ generates the connected functions. The angular brackets denote a thermal average or a vacuum expectation value depending on whether the thermal effects of the soft modes (momenta $\sim g T$ ) are to be included or not. We now derive $Z[\eta, \alpha]$ with respect to $\alpha$ and take the time-derivative. As is well known, this leads to a term involving the commutators and a term involving the time-derivative of the current $I_{+}^{a}$. This can be simplified using the equations of motion and the operator algebra (17). The result is

$$
\begin{aligned}
\left(\partial_{0}+Q_{i} \mathcal{D}_{i}\right)\left\langle I^{a}(x, \vec{Q})\right\rangle & +f^{a b c}\left(\sqrt{\frac{4 \pi}{k}} \alpha^{b}\left\langle I^{c}\right\rangle+Q_{i}\left\langle A_{i}^{b}(x) I^{c}(x, \vec{Q})\right\rangle\right) \\
& +\sqrt{\frac{k}{4 \pi}} Q_{i} \partial_{0}\left\langle A_{i}^{a}\right\rangle-\left(Q_{i} \mathcal{D}_{i} \alpha\right)^{a}=0
\end{aligned}
$$

where

$$
\begin{aligned}
\left\langle I^{a}(x, \vec{Q})\right\rangle & =\left(-i \frac{\delta}{\delta \alpha^{a}(x, \vec{Q})}\right) W \\
\left\langle A_{i}^{b}(x) I^{c}(y, \vec{Q})\right\rangle & =\left(-i \frac{\delta}{\delta \eta_{i}^{b}(x)}\right)\left(-i \frac{\delta}{\delta \alpha^{c}(y, \vec{Q})}\right) W, \text { etc. }
\end{aligned}
$$


The sources are not yet set to zero in equations (19,20). Further, $\mathcal{D}_{i}^{a c}=\partial_{i} \delta^{a c}+f^{a b c}\left\langle A_{i}^{b}\right\rangle$. In a similar way, we can derive an equation for the quantity $\left\langle A_{I}^{a}(x)\right\rangle$. This is given by

$$
\frac{\partial^{2}}{\left(\partial x^{0}\right)^{2}}\left\langle A_{i}^{a}(x)\right\rangle+\left(\mathcal{D}_{j} \mathcal{F}_{i j}\right)^{a}+\Delta_{i}^{a}+\left[\sqrt{\frac{k}{4 \pi}} \int d \Omega Q_{i}\left(\alpha^{a}-2\left\langle I^{a}\right\rangle\right)\right]-\eta_{i}^{a}=0
$$

where

$$
\begin{aligned}
\Delta_{i}^{a}=f^{a b c}\left[\partial_{j}\left\langle A_{i}^{b}(x) A_{j}^{c}(x)\right\rangle\right. & +\left\langle A_{j}^{b}\left(\partial_{i} A_{j}^{c}-\partial_{j} A_{i}^{c}\right)\right\rangle+f^{c r s}\left(\left\langle A_{j}^{b}(x) A_{i}^{r}(x)\right\rangle\left\langle A_{j}^{s}(x)\right\rangle\right. \\
& +\left\langle A_{j}^{b}(x) A_{j}^{s}(x)\right\rangle\left\langle A_{i}^{r}(x)\right\rangle+\left\langle A_{j}^{b}(x)\right\rangle\left\langle A_{i}^{r}(x) A_{j}^{s}(x)\right\rangle \\
& \left.\left.+\left\langle A_{j}^{b}(x) A_{i}^{r}(x) A_{j}^{s}(x)\right\rangle\right)\right]
\end{aligned}
$$

and $\mathcal{F}_{i j}^{a}=\partial_{i}\left\langle A_{j}^{a}\right\rangle-\partial_{j}\left\langle A_{i}^{a}\right\rangle+f^{a b c}\left\langle A_{i}^{b}\right\rangle\left\langle A_{j}^{c}\right\rangle$.

Any correlation function involving the fields can be calculated using the equations (19-22). It is, of course, possible to write functional integrals for the correlation functions. However, since the currents of the WZNW-theory are the important quantities for calculating the correlators of the fields $A_{i}^{a}$, the present approach of obtaining differential equations using the Hamiltonian framework is somewhat simpler. Equations (19-22) are written without the renormalization factors; eventually, each average of an independent opertor product should have such a factor.

As an application of these equations, we calculate the correlator $\left\langle I^{a}(x, \vec{Q}) I^{b}\left(y, \vec{Q}^{\prime}\right)\right\rangle$ to the lowest order (with no loops). Differentiating the equations $(19,21)$ with respect to $\alpha^{b}\left(y, \vec{Q}^{\prime}\right)$ and setting all sources to zero, (indicated by a subscript zero on the angular average signs), we get

$$
\begin{aligned}
{\left[\left(\partial_{0}^{2}-\partial_{k} \partial_{k}\right) \delta_{i j}\right.} & \left.+\partial_{i} \partial_{j}\right]\left\langle A_{j}^{a}(x) I^{b}\left(y, \vec{Q}^{\prime}\right)\right\rangle_{0} \\
+\sqrt{\frac{k}{4 \pi}}\left[-i Q_{i}^{\prime} \delta^{a b} \delta(x-y)-2 \int d \Omega^{\prime \prime} Q_{i}^{\prime \prime}\left\langle I^{a}\left(x, \vec{Q}^{\prime \prime}\right) I^{b}\left(y, \vec{Q}^{\prime}\right)\right\rangle_{0}\right] \approx q & \\
\left(\partial_{0}+Q_{i} \partial_{i}\right)\left\langle I^{a}(x, \vec{Q}) I^{b}\left(y, \vec{Q}^{\prime}\right)\right\rangle_{0} & +\sqrt{\frac{k}{4 \pi}} \partial_{0}\left\langle A_{i}^{a}(x) I^{b}\left(y, \vec{Q}^{\prime}\right)\right\rangle_{0} \\
& +i\left(Q_{i} \partial_{i}\right) \delta^{a b} \delta(x-y) \delta\left(\vec{Q}, \vec{Q}^{\prime}\right) \approx 0
\end{aligned}
$$


We have dropped the higher loop terms, as emphasized by the approximate equality. Also we note that it is consistent to take $\left\langle I^{a}\right\rangle=0$. Equation (23) can be solved by using the Green's function

$$
G_{i j}(x, y)=\int \frac{d^{4} p}{(2 \pi)^{4}} e^{-i p(x-y)} \frac{i}{p^{2}}\left(\delta_{i j}-\frac{p_{i} p_{j}}{p_{0}^{2}}\right)
$$

The integrand is singular as $p_{0} \rightarrow 0$, indicating the residual gauge invariance under timeindependent transformations. Nevertheless we can use this in the usual way with some prescription for handling the singularity such as the $\alpha$-prescription [9]. Using the solution of $(23)$ in $(24)$ we get the equation

$$
\begin{array}{r}
\int d^{4} z d \Omega^{\prime \prime}\left[\left(\partial_{0}+Q_{i} \partial_{i}\right) \delta(x-z) \delta\left(\vec{Q}, \vec{Q}^{\prime \prime}\right)+\frac{i k}{2 \pi} \partial_{0}\left(Q_{i} G_{i j}(x, z) Q_{j}^{\prime \prime}\right)\right]\left\langle I^{a}\left(z, \vec{Q}^{\prime \prime}\right) I^{b}\left(y, \vec{Q}^{\prime}\right)\right\rangle \\
=\delta^{a b}\left[-i Q_{i} \partial_{i} \delta(x-y) \delta\left(\vec{Q}, \vec{Q}^{\prime}\right)+\frac{k}{2 \pi} \partial_{0}\left(Q_{i} G_{i j}(x, y) Q_{j}^{\prime}\right)\right] \quad(26)
\end{array}
$$

The solution to this equation is given by

$$
\begin{gathered}
\left\langle I^{a}(x, \vec{Q}) I^{b}\left(y, \vec{Q}^{\prime}\right)\right\rangle=\delta^{a b} \int \frac{d^{4} p}{(2 \pi)^{4}} e^{-i p(x-y)} F\left(p, \vec{Q}, \vec{Q}^{\prime}\right) \\
F\left(p, \vec{Q}, \vec{Q}^{\prime}\right)=f_{1} \delta\left(\vec{Q}, \vec{Q}^{\prime}\right)+f_{2}\left(\vec{Q} \cdot \vec{Q}^{\prime}-\frac{\vec{p} \cdot \vec{Q} \vec{p} \cdot \vec{Q}^{\prime}}{p_{0}^{2}}\right)+f_{3} \frac{\vec{p} \cdot \vec{Q} \vec{p} \cdot \vec{Q}^{\prime}}{p_{0}^{2}} \\
f_{1}=\frac{i \vec{p} \cdot \vec{Q}}{\left(p_{0}-\vec{p} \cdot \vec{Q}\right)} \\
f_{2}=i \sigma \frac{1}{\left(p_{0}-\vec{p} \cdot \vec{Q}-\frac{2}{3} \sigma\right)}\left(\frac{p_{0}+\vec{p} \cdot \vec{Q}}{p_{0}-\vec{p} \cdot \vec{Q}}\right) \\
f_{3}=-\frac{2}{3} \sigma \frac{p^{2}}{p_{0}^{2}} \frac{1}{\left(p_{0}-\vec{p} \cdot \vec{Q}-\frac{2}{3} \sigma\left(\frac{p^{2}}{p_{0}^{2}}\right)\right)} \\
\sigma=\frac{k}{4 \pi} \frac{p_{0}}{p^{2}}
\end{gathered}
$$

We have used the vacuum average. A thermal distribution for the soft modes can be taken into account by imposing periodicity in imaginary time. The correlator (27) is useful, for instance, in calculating the scattering of a gluon in the quark-gluon plasma. It is what defines the gluonic structure functions of the plasma. 
We now turn to a slightly different topic. The Hamiltonian analysis and the equations of motion can be considerably simplified for the longitudinal modes. Eventhough the restriction to just the longitudinal modes is not physically well motivated, the simplifications are interesting and could eventually be incorporated in a more general framework. For this reason, we shall briefly discuss the reduction to longitudinal modes.

The longitudinal modes can be parametrized in terms of a matrix field $U$ as

$$
A_{i}=-\partial_{i} U U^{-1}
$$

$U$ is in general time-dependent and hence the gauge potential (30) is not a pure gauge. The magnetic field, however, is zero. The electric field can be parametrized in terms of a field $\Pi$ as $E_{i}=-U\left(\partial_{i} \Pi\right) U^{-1}$. We can also define currents

$$
j_{ \pm}=U^{-1} J_{ \pm} U
$$

$j_{ \pm}$and $\Pi$ are gauge invariant degrees of freedom; the field $\Pi$ is constrained in terms of $j_{ \pm}$ by the Gauss law. Since $J_{ \pm}$commute with $A_{i}$, they obviously commute with $U$. Using this fact, the operator algebra can be simplified. The nontrivial commutators become

$$
\begin{aligned}
{\left[\partial_{i} \partial_{i} \Pi^{a}(x), \partial_{j} \partial_{j} \Pi^{b}(y)\right]=} & i f^{a b c} \partial_{k} \partial_{k} \Pi^{c}(x) \delta(\vec{x}-\vec{y}) \\
{\left[-\partial_{i} \partial_{i} \Pi^{a}(x), j_{ \pm}^{b}(y, \vec{Q})\right]=} & -i f^{a b c} j_{ \pm}^{c}(x, \vec{Q}) \delta(\vec{x}-\vec{y}) \\
& \pm \frac{i k}{4 \pi} \delta^{a b} Q_{i} \partial_{i} \delta(\vec{x}-\vec{y}) \\
{\left[j_{ \pm}^{a}(x, \vec{Q}), j_{ \pm}^{b}\left(y, \vec{Q}^{\prime}\right)\right]=} & i f^{a b c} j_{ \pm}^{c}(x, \vec{Q}) \delta(\vec{x}-\vec{y}) \delta\left(\vec{Q}, \vec{Q}^{\prime}\right) \\
\mp & \frac{i k}{4 \pi} \delta^{a b} Q_{i} \partial_{i} \delta(\vec{x}-\vec{y}) \delta\left(\vec{Q}, \vec{Q}^{\prime}\right)
\end{aligned}
$$

The Hamiltonian becomes

$$
\mathcal{H}=\int d^{3} x\left[\frac{1}{2}\left(\partial_{i} \Pi^{a} \partial_{i} \Pi^{a}\right)+\frac{2 \pi}{k} \int d \Omega\left(j_{+}^{a} j_{+}^{a}+j_{-}^{a} j_{-}^{a}\right)\right]
$$

The Hamiltonian does not involve the field $U$ directly and hence the commutator of $\Pi^{a}$ with $U$, which is nontrivial, is not needed to analyze the dynamics of the longitudinal modes. 
The Gauss law condition becomes

$$
-\partial_{i} \partial_{i} \Pi^{a}+\int d \Omega\left(j_{+}^{a}+j_{-}^{a}\right)=0
$$

The commutation rules (32) are consistent with imposing the Gauss law as a strong operator condition. We may thus take (34) as the definition of $\Pi^{a}$ and the Hamiltonian and the commutation rules (32c) define the dynamics, with one constraint, viz. $j_{+}^{a}(x-\vec{Q})-j_{-}^{a}(x, \vec{Q})=0$.

The Hamiltonian equations of motion are now

$$
\partial_{0} C_{1}^{a}-Q_{i} \partial_{i} C_{0}^{a}+f^{a b c} C_{0}^{b} C_{1}^{c}=0
$$

where $j_{+}^{a}=\frac{k}{4 \pi} C_{1}^{a}, C_{0}^{a}=\Pi^{a}-C_{1}^{a}$. This equation can also be written as

$$
\left(\partial_{0}+Q_{i} \partial_{i}\right) C_{1}^{a}+f^{a b c} \Pi^{b} C_{1}^{c}=Q_{i} \partial_{i} \Pi^{a}
$$

We have a similar equation for $C_{1}^{\prime a}=\frac{4 \pi}{k} j_{-}^{a}$, obtained from (36) by $\vec{Q} \rightarrow-\vec{Q}$. The Gauss law is

$$
-\partial_{i} \partial_{i} \Pi^{a}+\frac{k}{4 \pi} \int d \Omega\left(C_{1}^{a}+C_{1}^{\prime a}\right)=0
$$

The solution of these equations, if we neglect the term involving the structure constants $f^{a b c}$, are the Abelian longitudinal plasma waves. Equation (36) can thus be used to systematically improve on the Abelian approximation.

\section{Appendix A}

As is well known, WZNW-theories for unitary matrices lead to a quantization requirement on the constant $k$ multiplying the action. This arises from the single-valuedness of the transition amplitudes or wavefunctions. For our case, eventhough the auxiliary field $G$ is a unitary matrix, there is no such requirement. The Wess-Zumino term which leads to the quantization requirement can be written, for a fixed choice of $Q_{i}$, as

$$
\mathcal{S}_{W Z}=\frac{1}{12 \pi} \int d^{2} x^{T} \int_{M^{3}} \operatorname{Tr}\left(d G G^{-1}\right)^{3}
$$


where $M^{3}$ is a space whose boundary is the two-dimensional world of the lightcone coordinates $x^{ \pm}$. $\mathcal{S}_{W Z}$ can be thought of as being obtained by integrating $\delta \mathcal{S}_{W Z}$ with the extra coordinate of $M^{3}$, say $s$, parametrizing the path of integration. Physical results are independent of this path. The difference for two extensions of the field $G$ into the extra dimension corresponding to $s$ is given by

$$
Q=\frac{1}{12 \pi} \int_{S^{3}} \operatorname{Tr}\left(d G G^{-1}\right)^{3}
$$

This is for the intrinsic two-dimensional problem, ignoring the transverse coordinates. $S^{3}$ is the three-dimensional sphere. $Q$ is an integer corresponding to the winding number of the map $G$ over the three-sphere. (The nontriviality of (A2) has to do with the fact that $\operatorname{Tr}\left(d G G^{-1}\right)^{3}$ is a closed but nonexact differential form. ) The single-valuedness of $e^{i k S_{W Z}}$ then requires $k$ to be an integer.

For our case, we have the two transverse coordinates as well; we can write equation (A1) as

$$
\mathcal{S}_{W Z}=\frac{1}{12 \pi} \int_{M^{5}} \operatorname{Tr}\left(d G G^{-1}\right)^{3} \omega
$$

where $\omega=\epsilon_{i j k} Q^{i} d x^{j} d x^{k}$. There is no restriction for the exterior derivatives in (A3) to be with respect to $x^{ \pm}$or $s$; this is enforced by the choice of $\omega$. Notice that eventhough $\operatorname{Tr}\left(d G G^{-1}\right)^{3}$ is not exact, the differential form in (A3) will be, if $\omega$ is exact. $\omega$ can be formally written as $d \rho$, but the one-form $\rho$ is linear in the coordinates and so we cannot use this unless we impose some stringent fall-off conditions on the fields $G$. However, when we take the difference of two extensions of $G$ into $M^{5}$, we get the quantity on the right hand side of (A3) integrated over a five-sphere. $\rho$ exists with no singularity and the integrand is exact. There is no nonzero winding number and no quantization of $k$.

\section{Appendix B}

The Hamiltonian for the effective action was obtained in ref.[5] by taking the derivative of the action with respect to the final value of time in a discretized version. This is a standard result, but does not seem to be well appreciated. A short argument justifying the calculation will be sketched here. 
We shall use the example of a scalar field to illustrate the calculation. Consider the Lagrangian

$$
L=\frac{1}{2}\left(\frac{\partial \phi}{\partial t}\right)^{2}-V(\phi)
$$

where $V(\phi)$ includes terms which do not depend on time-derivatives of $\phi$. The Hamiltonian, by definition, is the generator of reparametrizations of the time-variable $t$. Let us consider therefore the infinitesimal transformation $t \rightarrow \tilde{t}=t+\epsilon(t)$, where $\epsilon(t)$ is a function of $t$. We then have $d \tilde{t}=d t\left(1+\epsilon^{\prime}\right), \partial_{\tilde{t}}=\left(1-\epsilon^{\prime}\right) \partial_{t}$, where the prime denotes differentiation with respect to $t$. For the variation of the action $S=\int d t L$, we then find

$$
\delta S=-\int d t \epsilon^{\prime} \int d^{3} x\left[\frac{1}{2}\left(\frac{\partial \phi}{\partial t}\right)^{2}+V(\phi)\right]+\int \mathcal{E} \delta \phi
$$

$\delta \phi$ is the change induced in $\phi$ and $\mathcal{E}$ is the variational derivative. (Setting $\mathcal{E}$ to zero will give the equations of motion.) The final limit of the time-integration is not changed in this variation, only the coordinate label for it is varied. From equation (B2) we can identify the Hamiltonian as

$$
H=\int d^{3} x\left[\frac{1}{2}\left(\frac{\partial \phi}{\partial t}\right)^{2}+V(\phi)\right]
$$

If one imposes the equation of motion, viz. $\frac{\delta S}{\delta \phi}=0$, one can prove the conservation of $H$. However, to identify the expression for $H$ we do not impose the equations of motion; we just use the separation of $\delta S$ in the form given in (B2).

We can recast the above result as follows. Let us write the integral over time by discretizing the time-interval, with $t_{N}$ being the final time-label. Then $\epsilon^{\prime}\left(t_{n}\right)=\frac{\epsilon_{n}-\epsilon_{n-1}}{t_{n}-t_{n-1}}$, etc. The action can be written as

$$
S=\int d^{3} x\left[\frac{1}{2} \sum \frac{\left(\phi_{n-i}-\phi_{n-i-1}\right)^{2}}{\left(t_{n-i}-t_{n-i-1}\right)}-(V(\phi))_{n-i}\left(t_{n-i}-t_{n-i-1}\right)\right]
$$

The expression (B2) for $\delta S$ can be written as

$$
\delta S=-\left[\left(\epsilon_{N}-\epsilon_{N-1}\right) H\left(t_{N}\right)+\ldots\right]+\int \mathcal{E} \delta \phi
$$

We see that $H$ can also be identified as the coefficient of $\epsilon_{N}$ in the first set of terms. However, $\epsilon_{N}$ is the shift in $t_{N}$; thus the coefficient of $\epsilon_{N}$ is the derivative of the action with 
respect to the final time-label $t_{N}$. However, in taking this derivative we should not vary the fields as functions of time so as to avoid contributions from the second set of terms, viz. those proportional to $\mathcal{E}$. This qualification means that we can use expression (B4) for the action, but interpret $\phi_{n-i}$ as independent variables, not as $\phi\left(t_{n-i}\right)$. Further, although we change $t_{N}$, the number of segments of time interval is fixed. The limits of the summation are unchanged, reflecting the fact that the variation in (B2) only changes the coordinate labels. Applied to the effective action, the above argument gives the Hamiltonian (12), i.e. the result in ref.[5].

\section{Appendix C}

In this appendix, we discuss the canonical derivation of the operator algebra.

The general method is as follows. Given an action $S$ depending on a set of fields $\varphi_{r}, r=1,2, \ldots n$, we consider its variation under a change of fields. This is of the form

$$
\delta S=\int d^{4} x \mathcal{E}_{r} \delta \varphi_{r}+\oint K_{\mu} d \sigma^{\mu}
$$

where $\mathcal{E}_{r}$ is the variational derivative. The second term is the surface contribution, with $K^{\mu}$ being linear in the variations $\delta \varphi_{r}$. If we consider the time-coordinate as the evolution parameter and impose suitable boundary conditions at spatial boundary (viz. that $K_{i}$ vanishes there), we can write the surface term as the difference of $\Theta$ 's at the initial and final time-slices, where

$$
\Theta=\int d^{3} x K_{0}
$$

$\Theta$ is the canonical one-form. The variation of $\Theta$, antisymmetrized in the variations, (i.e. the exterior derivative on the field space) gives the symplectic structure $\omega$. (This is an old result going back to nineteenth century analytical mechanics; in the context of quantum field theory, Schwinger's action principle is closely related to this. For some modern references, see [10]. Also, an analogous method can be used even if the initial and final data hypersurfaces do not correspond to constant time-slices.) There are ambiguities in identifying $\Theta$ as above, since we can add total divergences to the Lagrangian; $\omega$ has no such ambiguity. (The changes in $\Theta$ given by such total divergences are, of course, the canonical transformations.) 
Given $\omega$, the Poisson brackets for the canonical coordinates are given by the inverse to $\omega_{i j}$, viewed as an antisymmetric matrix, where $\omega=\frac{1}{2} \omega_{i j} \delta \sigma^{i} \delta \sigma^{j}$, and $\sigma^{i}$ denote the canonical variables (like the fields or their time-derivatives). The commutators are then $i$ times the Poisson brackets. For the present problem, we need commutators for currents, rather than the canonical coordinates, and for this purpose it is algebraically simpler to use an equivalent definition. Given $\omega$, a vector field $\xi_{A}$ is said to be Hamiltonian if $\left.\xi_{A}\right\rfloor \omega=-\delta A$, for some function $A$; alternatively, given any function $A$, we can associate a vector field to it by this condition. The notation is as follows. If we write $\omega$ in terms of its antisymmetric components $\omega_{i j}$ and $\xi=\xi^{i} \frac{\delta}{\delta \sigma^{i}}$, then

$$
\xi\rfloor \omega=\xi^{i} \omega_{i j} \delta \sigma^{j}
$$

The commutator of two functions $A$ and $B$ is given by

$$
\left.\left.[A, B]=i\left(\xi_{A}\right\rfloor \xi_{B}\right\rfloor \omega\right)
$$

This method can be applied to our problem. The only technical complication is that the WZ-term requires an extension of the fields into an extra dimension. We can take the extended space $M^{3}$ as $M^{2} \times[0,1], s=1$ being real spacetime. The variations of the fields at $s=0$ can be taken to be zero. From the variation of the effective action and after identifying $\Theta$, the symplectic structure can be obtained as

$$
\begin{array}{r}
\omega=\int d^{3} x \delta E_{i}^{a} \delta A_{i}^{a}+\frac{k}{4 \pi} \int d^{3} x d \Omega \operatorname{Tr}\left[v \delta L_{0}+v^{2}\left(L_{0}+Q_{i} L_{i}\right)\right. \\
\left.+v Q_{i} \delta A_{i}+G^{-1} v G Q_{i} \delta A_{i}\right]
\end{array}
$$

where $v=\delta G G^{-1}, L_{\mu}=\partial_{\mu} G G^{-1}, R_{\mu}=G^{-1} \partial_{\mu} G$. The Hamiltonian vector fields are

$$
\xi_{A}=-\frac{\delta}{\delta E_{i}^{a}}, \quad \xi_{E}=\frac{\delta}{\delta A_{i}^{a}}-\int d \Omega Q_{i}\left(\frac{\delta}{\delta L_{0}^{a}}+\frac{\delta}{\delta R_{0}^{a}}\right)
$$

For the variation $\delta G=\theta G$, the vector field is

$$
\xi_{\theta}=V(\theta)+\int d^{3} x d \Omega \frac{k}{8 \pi} Q_{i} \theta^{b}\left(\delta^{b a}-2 M^{b a}\right) \frac{\delta}{\delta E_{i}^{a}}
$$


where $M^{a b}=\operatorname{Tr}\left[\left(-i t^{a}\right) G\left(-i t^{b}\right) G^{-1}\right]$ and $V(\theta)$ replaces $v$ by $\theta$ and $\delta L_{0}$ by $\left[\theta, L_{0}+Q_{i} L_{i}\right]$. In other words,

$$
V(\theta)\rfloor \omega=\frac{k}{4 \pi} \operatorname{Tr}\left[\theta\left(\delta L_{0}+Q_{i} \delta A_{i}+G Q_{i} \delta A_{i} G^{-1}\right)\right]
$$

We can calculate the various commutators using these vector fields and the formula (C4). The result is the algebra (17) in the text.

\section{References}

[1] R. Pisarski, Physica A 158, 246 (1989); Phys.Rev.Lett. 63, 1129 (1989); E. Braaten and R. Pisarski, Phys.Rev. D 42, 2156 (1990); Nucl.Phys. B 337, 569 (1990); ibid. B 339, 310 (1990); Phys.Rev. D 45, 1827 (1992); J. Frenkel and J.C. Taylor, Nucl.Phys. B 334, 199 (1990); J.C. Taylor and S.M.H. Wong, Nucl.Phys. B 346, 115 (1990).

[2] R. Efraty and V.P. Nair, Phys.Rev.Lett. 68, 2891 (1992); Phys.Rev D 47, 5601 (1993).

[3] R. Jackiw and V.P. Nair, Phys.Rev. D 48, 4991 (1993)

[4] J.P. Blaizot and E. Iancu, Phys.Rev.Lett. 70, 3376 (1993); Saclay preprint T93/064, to appear in Nucl.Phys. B.

[5] V.P. Nair, Phys.Rev. D 48, 3432 (1993).

[6] R. Jackiw, Q. Liu and C. Lucchesi, MIT preprint CTP\#2261 (1993); J.P. Blaizot and E. Iancu, Saclay preprints T94/02,03, 013 (1994).

[7] S.P. Novikov, Usp.Mat.Nauk 37, 3 (1982); E. Witten, Commun.Math.Phys. 92, 455 (1984).

[8] R.I. Nepomechie, Phys.Rev. D 33, 3670 (1986); D. Karabali, H.J. Schnitzer and Z. Yang, Phys.Lett. 216 B, 307 (1989); D. Karabali and H.J. Schnitzer, Nucl.Phys. B 329, 649 (1990); K. Gawedzki and A. Kupianen, Phys.Lett. 215 B, 119 (1988); Nucl.Phys. B 320, 649 (1989).

[9] see for example, P.V. Landshoff, Nucl.Phys. B Proc.Suppl. 16, 597 (1990). 
[10] V. Guillemin and S. Sternberg, Symplectic Techniques in Physics, Cambridge University Press (1990); J. Schwinger, Phys.Rev. 82, 914 (1951); C. Crnkovic and E. Witten, in Three Hundred Years of Gravitation, S.W. Hawking and W. Israel (eds.), Cambridge University Press (1987); G.J. Zuckerman, in Mathematical Aspects of String Theory, S.T. Yau (ed.), World Scientific (1987). 